\title{
Cervical cancer and human papilloma virus (HPV) - viral cervical cancer
}

\begin{abstract}
Cervical cancer is ranking second among women worldwide. The most important viral causative agent of cervical cancer is recognized as Human Papilloma viruses. Because of defect or loss of modern and advanced diagnostic tools, and proper screening assays the number of cervical cancer and related mortality is still high. In this review article, cervical cancer epidemiology, General properties of HPV and the life cycle, and HPV Genomics as a new pathway for advanced molecular diagnostic have been discussed.

Regarding to preparing this review, the Google Scholar search engine and PubMed database were applied for selecting the appropriate review and original articles. The searched articles were studied and evaluated to employ them as invaluable scientific sources.

The most common way for transmitting HPVs cervical cancer is sexual activities. Persistent of the HPV infections in cervix area leads to cervical cancer. In recent decades, the progression of molecular diagnostics was considerable. Today, there are a huge data about molecular biology and genomic characterizations pertaining to HPVs. Advanced molecular technologies like microarray may be a good substitution for routine diagnostics. Progression in modern molecular approaches needs a great knowledge about HPVs genomic properties.

In conclusion, microarray as a rapid, accurate and reliable diagnostic technology will enable us to have a desirable diagnostic tool in association to HPV cervical cancers within early stages. This may lead to decrease of progressive cervical cancers and high rate of mortality.
\end{abstract}

Keywords: human papilloma virus, cervical cancer, genomicss
Volume 2 Issue 3 - 2015

\author{
Payam Behzadi,' Elham Behzadi, ${ }^{2}$ Reza \\ Ranjbar,' Esmaiel Saberfar ${ }^{3}$ \\ 'Molecular Biology Research Center, Baqiyatallah University of \\ Medical Sciences, Tehran, Iran \\ ${ }^{2}$ Microbiology department, Islamic Azad University, Shahr-e- \\ Qods Branch, Tehran, Iran \\ ${ }^{3}$ Applied Virology Research Center, Baqiyatallah University of \\ Medical Sciences, Tehran, Iran
}

Correspondence: Reza Ranjbar, Molecular Biology Research Center, Baqiyatallah University of Medical Sciences, Tehran, Iran, Tel +98 2188039883 , Fax +98 2188039883 ,

Email ranjbarre@gmail.com

Received: April 18, 20I5 | Published: May 28, 2015
Abbreviations: HPV, human papilloma virus; HIV, human immunodeficiency virus; IARC, international agency for research on cancer; PCRs, polymerase chain reactions; ORF, open reading frames; LCR, long control region

\section{Introduction}

Cancers are known as the most deathful diseases which threat the health of human beings. Among different types of cancers, breast cancer ranks first and the cervical cancer stands in second place among women populations around the world. On the other hand, 15\% of human cancer cases are in association with viruses. $50 \%$ of human viral cancers are caused by Human Papilloma virus (HPV). ${ }^{1-3}$

The genomic maps show three different types of genital, nongenital and verruca producer types of HPVs. HPVs are recognized as species specific viruses and the human cells are their single known reservoir. $^{4}$

The HPV malignancies are the commonest among different vira sexual transmitted infections and diseases. There are two types of HPVs, low risk and high risk HPVs. The high risk types of HPVs are considered as viral pathogens which may cause cervical cancers and malignancies resulted in the persistence of infection. ${ }^{1-3}$

In addition to cervix, other anatomical parts of female's body including anus, vagina and vulva may be involved by oncogenic HPV. The HPV oncogenesis is directly related to epithelial and mucosa tropism attitude of the virus. ${ }^{5}$
Many women suffer from cervical cancer as a viral infectious disease. A numerous number of cervical cancers are caused by HPV which may lead to death. The persistent HPV infections increase the risk of cervical cancer. The high level of mortality among women with cervical cancer, has led to a huge concern to control and prevent cervical cancer in association with HPV worldwide. However, the majority of HPV infections disappears by the time and is not dangerous. ${ }^{1,5-8}$

There are several routine viral diagnostics including cytological (Pap smear), histopathological and some conventional PCR assays which are not rapid and precise. The use of molecular biology and genetics has led to new advanced molecular diagnostic approach like microarray technologies. ${ }^{1,9,10}$

As HPV is a considerable viral agent which causes genital malignancies and cancers, a rapid and accurate diagnostic method like microarray is necessary. To provide advanced molecular diagnostics, there is an essential need to HPVs genomics. ${ }^{1,11-13}$ Furthermore, knowledge about HPVs cervical cancer epidemiology, general properties of HPV and the life cycle describe the importance of HPVs infections and diseases. Thus, this review article has a look on aforementioned attitudes of HPV and cervical cancer.

\section{Epidemiology}

According to previous investigations, the rate of HPV cervical cancer and the related deaths in Africa (North African countries are excluded), South America, and South-Central Asian countries 
is the highest. So, knowing the incidence rate of diseases may help to control and prevent infections. Totally, the global prevalence of cervical cancer among young and elder women is about $14 \% 0^{3,5}$

The commonest way for transmission of HPV infections is reported as sexual activities. Thus, females with high sexual activities and several sexual partners significantly increase the risk of cervical cancer. The incidence of HPV related cervical cancers is reported from different regions around the world; but it happens for the most in developing countries; because of their poor screening systems in public health care systems. ${ }^{1,5,6}$

The first identification regarding to HPV related cervical cancer was achieved by German virologist named Harold zur Hausen in 1980s. From this time, the oncogenic HPV is known as the most important viral agent that causes cervical cancer via producing carcinoma in cervical squamous tissues. ${ }^{5,8}$

Mostly, HPV infections occur in high sexual active women ranging from 18-30years old. However, cervical cancer happens in elder women with more than 35 years old because of the persistent of HPV infection regarding to oncogenic types. Generally, persistent HPV infections are revealed in about two years; however, the majority of HPV infections are removed by the human immune system after a period of 18 months. ${ }^{1,2,8,14}$

In addition to sexual activity, smoking, long time consumption of oral contraceptive, HIV infection, and Chlamydia infection are the most important predisposing factors which may lead to cervical cancers and malignancies. ${ }^{4,15}$

The immune system cell mediated pathway reacts against HPV infection as the first immune response; therefore, patients with human immunodeficiency virus (HIV) are not able to eliminate the viral agent of HPV. ${ }^{8}$

Researches show that over 200 types of HPV are existed. But only 40 types of them are responsible for serious human genital cancers. ${ }^{8,16}$

\section{General properties of HPV and the life cycle}

HPVs are belonging to the viral family of Papovaviridae. HPVs are small viruses with a size up to $60 \mathrm{~nm}$ in diameter encompass a capsid with icosahedral structure but these viruses miss envelop within their viral structure. The lack of envelope may lead to occurrence of resistant HPVs against antiviral agents. The life cycle of HPV is completed throughout the differentiation of cervical mucosa cells According to previous studies, the expression of L1 and L2 which are known as capsid proteins occurs on the surface of genital mucosa cells. The place of aforementioned proteins expression and viral assemblage are the same. By the proliferation of viral structures, the virion particles regarding to 8 -kilo base pair (8kbp) double stranded circular DNA viruses of HPVs are shed from the infected mucosa cells. The viral DNA has the ability of integration into the human cell DNA accidentally. The life cycle of HPVs is estimated up to 21 days. ${ }^{1,4,5,8,12,15,17}$

According to several previous investigations, cervix employs especial mechanisms concerning physical stresses and healing functional responses. Wounds in epithelial tissues of cervix are appropriate entries for HPVs to access the basal cells. The viral ulcers and lesions involve HPVs genomes within their cell components. Besides, HPVs are able to escape from cervical healing system. The physiologic conditions of cervix support the persistence of HPVs. Moreover, in the middle of squamous epithelial tissues located within the ectocervix zone and the columnar epithelial tissues situated in endocervix; there is a transformation region which is an ideal zone for initiation and progression of HPVs cervical cancer. The differentiation ability in basal cells in transformation region of cervix makes a positive tropism for HPVs. The proliferation of HPVs and the related virion particles is dependent on basal cells differentiation. The presence of hormones including progesterone and estrogen induce viral infection and cervical cancer as orchestrating system in the cervical ecosystem. ${ }^{2,4,18,19}$

The molecular biologic surveys indicate that HPVs within the basal cells undergo the DNA replication which leads to appearance of over thousands copies of DNA within each cell. This process induces the expression of late genes which provide the release of virions and other viral particles. ${ }^{12,17}$

HPVs are classified into 5 main genera including $\alpha$-types (with genital tropism), $\beta$-types (with cutaneous tropism), $\gamma$-types, $\mu$ types and $v$ types. The oncogenic mucosal HPVs which are categorized as $\alpha$-types involve high risk HPVs and low risk HPVs. The high risk HPVs are responsible for cervical cancer. ${ }^{8,15,17,20,21}$

According to International Agency for Research on Cancer (IARC), the high risk HPVs include 12 types of 82, 73, 68, 59, 58, 56, $52,51,45,39,35,33,31,18$, and 16 while the low risk HPVs consist $81,72,70,61,54,44,43,42,40,11$, and $6 .^{1,15,17}$

The most frequent HPV types which may lead to cervical cancer are recognized as HPV16 ( $50 \%$ of cases) and HPV18 $(20 \%$ of cases). HPV16 is able to escape professionally from human immune system response in genital tract. That is why; the persistent of HPV16 causes cervical cancer and malignancies for the most. Genetic data indicate that, the low risk HPVs DNA molecules are not integrated into the host cell's DNA, while the high risk HPVs and in particular HPV16 and HPV18 integrate their DNA molecules into the host cell's DNA; thus, the viral DNA integration may cause cervical cancers and malignancies. ${ }^{4,17}$

The HPV malignancies develop throughout the persisted viral lesions. As the viral lesions progress by the time, a considerable need for nutrition and oxygen is observed among infected cervical cells which in consequence it may lead to cell death. The unsuitable condition within infected cells will be controlled via the feature of angiogenesis. Angiogenesis is triggered via HPVs proteins which are produced by the viral genes. The activation of angiogenesis is a vital phenomenon for HPVs proliferation and persistence within infected cervical cells. ${ }^{2,13,22,23}$

\section{HPV genomics}

The ubiquitous viral agents of Papilloma viruses (PVs) have a 350 million-year-old relationship with reptiles as their first hosts. But, they have evolved by the time and today, humans are included as their other hosts. The phylogenetic investigations have revealed that HPVs have been evolved together with their human host step by step. This viral attribute makes it a causative agent of infections with invisible and chronic properties. ${ }^{11,12}$

A huge portion of genomic secrets and enigmas regarding to HPVs is dissolved via molecular techniques. The initial genetic discoveries and identification of several genes and gene expression was achieved throughout the Polymerase Chain Reactions (PCRs). A portion of molecular mechanisms of HPV biology is in association with PCR technologies. ${ }^{11}$ 
The evolved HPVs genomes have undergone several mutations and rearrangements over the millennia. $\alpha$-HPV types encompass a larger genome in comparison with $\beta$-HPV types. The HPVs genomes possess up to 9 open reading frames (ORF) with a determined diversity on the similar DNA molecule. The general structure of HPV genome involves three zones of coding and upstream regulated non-coding regions. The coding zones are categorized into two parts of early gene coding zone and late gene coding zone. ${ }^{17,24}$

Table I Functional proteins recognized in $\alpha-H P V$ types
Early gene coding zone embraces six ORFs of E5, E4, E2, E1, and E7, E6. Late gene coding zone includes L1 and L2 ORFs which encode major and minor capsid proteins respectively. And non-coding zone situated in the separating boards of L1 and E6 start codons which is comprised of long control region (LCR) with the ability of regulatory functions in association with HPV replication and transcription (Table 1) (Figure 1). ${ }^{2,8,11,12,17}$

\begin{tabular}{|c|c|c|c|c|c|c|c|}
\hline \multicolumn{8}{|c|}{ Functional HPV proteins } \\
\hline LI & L2 & E I & E2 & E4 & E5 & E6 & E7 \\
\hline $\begin{array}{l}\text { Minor component } \\
\text { of HPV capsid } \\
\text { protein, type } \\
\text { specific epitope }\end{array}$ & $\begin{array}{l}\text { Major component } \\
\text { of HPV capsid } \\
\text { protein, group } \\
\text { specific epitope }\end{array}$ & $\begin{array}{l}\text { Contributes } \\
\text { in HPV DNA } \\
\text { replication }\end{array}$ & $\begin{array}{l}\text { Collaboration with EI in viral } \\
\text { DNA replication, regulation } \\
\text { of early genes expression } \\
\text { (particularlyE6 and E7) }\end{array}$ & $\begin{array}{l}\text { Contribution } \\
\text { in virion } \\
\text { release }\end{array}$ & $\begin{array}{l}\text { Viral } \\
\text { evade }\end{array}$ & $\begin{array}{l}\text { Oncogenesis, } \\
\text { genome } \\
\text { amplification }\end{array}$ & $\begin{array}{l}\text { Oncogenesis, } \\
\text { genome } \\
\text { amplification }\end{array}$ \\
\hline
\end{tabular}



Figure I Human papilloma virus type 6 complete genome, the numbers mentioned in this figure is in base pair. ${ }^{27}$

Molecular biology and Genomics relating to HPVs have revealed the viral reproductive genetic tactics, the type of viral genetic adaptation, the structure of viral genome etc. Simultaneously, the use of molecular diagnostic tools is helpful in molecular epidemiology. Via molecular epidemiology, the phylogenetic characterizations pertaining to each type of HPV are determined. ${ }^{11}$

Phylogentic analyses show the importance of L1 gene for HPVs detection and identification via nucleic acid based diagnostics. L1 gene owns a high level conserved sequence; therefore it is recognized as an appropriate standard criterion for genetic typing. This occurrence is an opportunity for providing advanced molecular diagnostic tools such as microarray technologies rather than PCRs. Microarray technologies enable us to create a huge gallery relating to gene and gene expression profiles regarding to HPVs using for clinical diagnoses. ${ }^{9-11,25,26}$

\section{Microarray technology}

Microarray technologies are advanced diagnostic methods which are able to use RNA and DNA molecules as target sequences. DNA technology is a progressed technique which employs fixed probes on the chip to detect or identify labeled DNA molecules. DNA microarray is a multistage diagnostic tool involving probe designing, spotting, DNA labeling, hybridization and scanning. The data received from scanner may be huge and abundant. But, the interpretation of the data is quite important. Today, there are several free databases which may be used to have an appropriate data interpretation. Furthermore, the use of suitable platforms and probe sets are effective to have a proper performance of DNA microarray technology as an invaluable advanced molecular diagnostic method. Microarray technology is a rapid, accurate, effective, multifunctional, sensitive and specific diagnostic method which can be used for detecting and identifying microbial agents such as HPV within clinical samples. DNA microarray is able to handle hundreds of thousands clinical samples in the same time. Besides, DNA microarray gives us the ability of gene and gene expression profiling for HPVs. Hence, microarray and in particular DNA microarray is a rapid and accurate diagnostic tool with high specificity and sensitivity. ${ }^{9,10,21,25,26,28}$

\section{Discussion}

HPVs are responsible for different cancers in women. Generally, the infections caused by HPVs are healed spontaneously and no cancer happens. But, there are some risk factors which predispose individuals for cervical cancer. High sexual activities and multi-sex partners are the most significant risk factors for cervical infections. The persistence of infection leads to cervical cancers. For this reason, people must be checked via viral diagnostic method. ${ }^{1,2,8,15,24}$

As there is no specific medium culture for HPV, cytological tests, histopathological assays and some conventional molecular diagnostics are performed for diagnosing samples to detect HPV high risk types. Routine diagnostic methods like Papanicaolaou test (Pap smear) providing results with the accuracy level of $80 \%$. But, this is not acceptable for the modern medical societies. All these tests are neither rapid nor $100 \%$ accurate. Besides, conventional PCRs are limited and time consuming. PCR technologies are effective when the number of clinical samples is low. Otherwise, PCR techniques are not cost effective and rapid when you have an abundance number of clinical specimens. ${ }^{1,924,26,29-31}$ 
The high risk HPVs are the etiologic agents that cause cervical cancer throughout persisting in cervical tissues within about 2 years. The main point is to detect the high risk HPVs in early stages of infections. A valuable diagnosis needs advanced and modern diagnostic tools. Microarray technologies are known as advanced and miniaturized molecular diagnostics which are rapid, accurate with high specificity and sensitivity. The use of DNA microarray enables us to have a rapid and effective diagnosis in our clinical samples. Detection of HPV cervical cancers within early stages with high specificity and sensitivity is guaranteed by microarray which may lead to decrease the mortality. ${ }^{910,21,25,26,28}$

Microarray technologies are cost effective, effective, rapid and accurate. Therefore, molecular biology and Genomics researches are helpful for finding new promises in the field of advanced molecular diagnostics. Gene profiling via microarray technology may be a good choice as progressive molecular diagnostic technology. ${ }^{1,9,10,21,25,28}$

\section{Conclusion}

Molecular biology and Genomics are two important pathways for providing rapid, accurate, specific and sensitive molecular diagnostics. In parallel with vaccination as a suitable prevention method, rapid and accurate diagnostics may be a good choice for decreasing the rate of cervical cancers around the world; even in poor and developing countries.

\section{Acknowledgements}

None.

\section{Conflict of interest}

The author declares no conflict of interest.

\section{References}

1. Behzadi P, Behzadi E, Ranjbar R, et al. Vaginal cancers and human papilloma virus. Oncolog Hematolog. 2015;30(1):14-18.

2. Bodily J, Laimins LA. Persistence of human papilloma virus infection: keys to malignant progression. Trends Microbiol. 2011;19(1):33-39.

3. Jemal A, Bray F, Center MM, et al. Global cancer statistics. $C A: A$ Cancer Journal for Clinicians. 2011;61(2):69-90.

4. Gearhart PA, Randall TC, Buckley RM, et al. Human Papilloma virus MedScape. 2015.

5. Crosbie EJ, Einstein MH, Franceschi S, et al. Human papilloma virus and cervical cancer. The Lancet. 2013;382(9895):889-899.

6. Lowy DR, Schiller JT. Reducing HPV-associated cancer globally. Cancer Prev Res (Phila). 2012;5(1):18-23.

7. Arbyn M, Castellsague X, De Sanjose S, et al. Worldwide burden of cervical cancer in 2008. Annals of oncology. 2011;22(12):2675-2686.

8. Burd EM. Human papilloma virus and cervical cancer. Clin Microbiol Rev. 2003;16(1):1-17.

9. Najafi A, Ram M, Ranjbar R. Microarray Principles \& Applications. 1st ed. Iran: Persian Science \& Research Publisher; 2012.

10. Behzadi P, Behzadi E, Ranjbar R. The application of Microarray in Medicine. ORLro. 2014;24(3):24-26.

11. Harari A, Chen Z, Burk R. Human papilloma virus genomics: past, present and future. Current problems in dermatology. 2014;45:1-18.
12. Doorbar J, Egawa N, Griffin H, et al. Human papilloma virus molecular biology and disease association. Reviews in medical virology. 2015;25(S1):2-23.

13. Hanahan D, Weinberg RA. The hallmarks of cancer. Cell.2000;100(1):5770 .

14. Chen AA, Heideman DA, Boon D, et al. Human papilloma virus 33 worldwide genetic variation and associated risk of cervical cancer. Virology. 2014;448:356-362.

15. Chelimo C, Wouldes TA, Cameron LD, et al. Risk factors for and prevention of human papilloma viruses (HPV), genital warts and cervical cancer. J Infect. 2013;66(3):207-217.

16. Sheikh N, Shaukat A, Javaid H. Human Papilloma Virus. Structure. 2014; $18: 7$.

17. Tommasino M. The human papilloma virus family and its role in carcinogenesis. Semin Cancer Biol. 2014;13-21.

18. Timmons B, Akins M, Mahendroo M. Cervical remodeling during pregnancy and parturition. Trends in Endocrinology \& Metabolism. 2010;21(6):353-361.

19. Iwasaki A. Antiviral immune responses in the genital tract: clues for vaccines. Nature Reviews Immunology. 2010;10(10):699-711.

20. Steben M, Duarte-Franco E. Human papilloma virus infection: epidemiology and pathophysiology. Gynecol Oncol. 2007;107(2):S2-S5.

21. Berthet N, Falguieres M, Filippone C, et al. Resequencing Microarray Technology for Genotyping Human Papilloma virus in Cervical Smears. PloS one. 2014;9(11):e109301.

22. Toussaint-Smith E, Donner DB, Roman A. Expression of human papilloma virus type $16 \mathrm{E} 6$ and E7 oncoproteins in primary foreskin keratinocytes is sufficient to alter the expression of angiogenic factors. Oncogene. 2004;23(17):2988-2995.

23. Smith-McCune KK, Weidner N. Demonstration and characterization of the angiogenic properties of cervical dysplasia. Cancer Res. 1994;54(3):800-804.

24. Burk RD, Harari A, Chen Z. Human papilloma virus genome variants. Virology. 2013;445(1-2):232-243.

25. Behzadi P, Behzadi E, Ranjbar R. Microarray Data Analysis. Alban Med J. 2014;4:84-90.

26. Shen-Gunther J, Rebeles J. Genotyping human papilloma viruses: development and evaluation of a comprehensive DNA microarray. Gynecol Oncol. 2013;128(3):433-441.

27. Human papilloma virus type 6 complete genome, isolate 126 [Internet].

28. Chepovetsky J, Kalir T, Weiderpass E. Clinical applicability of microarray technology in the diagnosis, prognostic stratification, treatment and clinical surveillance of cervical adenocarcinoma. Current pharmaceutical design. 2013;19(8):1425-1429.

29. Dhakal HP, Pradhan M. Histological pattern of gynecological cancers Journal of Nepal Medical Association. 2009;48(176).

30. Verma I, Jain V, Kaur T. Application of Bethesda System for Cervical Cytology in Unhealthy Cervix. Journal of clinical and diagnostic research: JCDR. 2014;8(9):OC26-OC30.

31. Porras C, Hildesheim A, González P, et al. Performance of Self-Collected Cervical Samples in Screening for Future Precancer Using Human Papilloma virus DNA Testing. J Natl Cancer Inst. 2015;107(1):dju400. 